\title{
A high-resolution rotation curve of NGC 6822
}

\author{
W. J. G de Blok \\ Dept of Physics and Astronomy, Cardiff University, 5 The Parade, \\ Cardiff, CF24 3YB, UK \\ D. T. F. Weldrake \\ RSAA, Mt Stromlo Observatory, Cotter Road, Weston, ACT 2611, \\ Australia \\ F. Walter \\ NRAO AOC, PO Box O, 1003 Lopezville Road, Socorro, NM \\ 87801-0387, USA
}

\begin{abstract}
We present a high-resolution rotation curve of the Local Group dwarf irregular NGC 6822, obtained with the Australia Telescope Compact Array. The best curve has a resolution of $8^{\prime \prime}$ or $20 \mathrm{pc}$ and contains some 250 independent points. NGC 6822 is a very dark matterdominated galaxy. There is no evidence for the presence of a steep density cusp down to scales of $\sim 20 \mathrm{pc}$, in conflict with the predictions of CDM.
\end{abstract}

NGC 6822 is the most nearby (490 kpc) late-type dwarf galaxy outside the Milky Way/LMC/SMC system and therefore a prime target to study in detail the distribution of dark and visible matter. Observations in the $21-\mathrm{cm}$ line of neutral hydrogen by de Blok \& Walter (2001) show that the galaxy is dark matter dominated, gas rich and reveals many interesting features such as a highly structured HI disk, a supergiant HI shell and a companion cloud. Here we discuss the dynamics and rotation curve of NGC 6822, and test various dark matter halo models.

NGC 6822 was observed with the Australia Telescope Compact Array with an angular resolution of $8^{\prime \prime}(20 \mathrm{pc})$ and a velocity resolution of $0.8 \mathrm{~km} \mathrm{~s}^{-1}$. The small distance and large angular size of the galaxy yielded a data set that has over 500 independent points along the major axis of the galaxy. We used a tilted-ring modeling program to derive the rotation curve of NGC 6822 .

Cosmological numerical Cold Dark Matter (CDM) simulations predict a specific and universal shape for the dark matter density distributions of galaxies. This was investigated in detail by Navarro, Frenk \& White $(1996,1997)$ [NFW] who found that the mass-density distribution in the inner parts of simulated CDM halos could best be described by a $r^{-1}$ power law. This is however not what is typically observed: the mass-density distributions of dwarfs and latetype galaxies, for example, are best described by an almost constant density central core $\rho(r) \sim r^{-0.2 \pm 0.2}$ (de Blok et al 2001). The NGC 6822 data enable us to measure the rotation curve of a dwarf galaxy an order of magnitude more accurately than the observations alluded to above, thus making NGC 6822 an ideal benchmark. 
We have fitted CDM NFW and constant density core pseudo isothermal models to the NGC 6822 rotation curve using the minimum disk assumption. These fits are presented in Fig. 1. The NFW model rotation curve can be described with two parameters: the collapse factor $c$ (which tells how much the original mass distribution has collapsed) and the typical rotation velocity $V_{200}$ (which is a proxy for the mass of the halo). These two parameters are related and their relation and values depend on the cosmology assumed. The pseudo-isothermal model (ISO) is described by a core-radius $R_{C}$ and a central density $\rho_{0}$. The panels in Fig. 1 compare the best-fit NFW and ISO models. It is clear that the NFW fit is much worse than the ISO fit. Also, the resulting fitting parameters are unphysical: $c$-values less than $\sim 2$ are not allowed in any CDM cosmology. Also shown is the best fitting model where we have taken cosmological constraints (i.e., we insist that this model fit conforms to what is expected in a $\Lambda$ CDM universe) into account by setting $V_{200}=V_{\max }$. The discrepancy between the observations and simulations is clear.

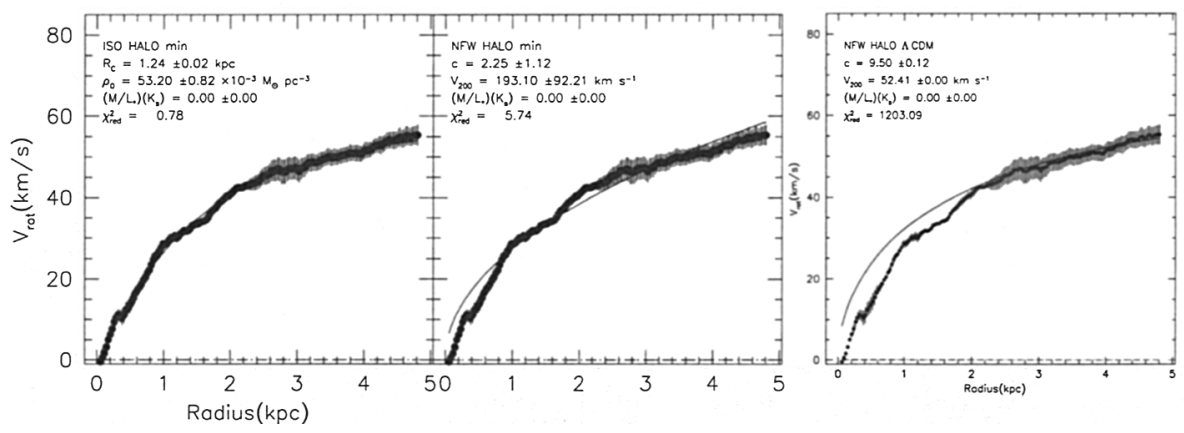

Figure 1. Best-fit models to NGC 6822 rotation curve. Left panel: ISO model. Middle panel: NFW model. Right panel: best fit cosmological CDM model, assuming $V_{200}=V_{\max }=52 \mathrm{~km} \mathrm{~s}^{-1}$.

\section{References}

de Blok, W. J. G., \& Walter, F. 2001, ApJ, 537, L95

de Blok, W. J. G., McGaugh, S. S., Bosma, A., \& Rubin, V. 2001, ApJ, 552, L23

Navarro, J. F., Frenk, C. S., \& White, S. D. M. 1996, ApJ, 462, 563

Navarro, J. F., Frenk, C. S., \& White, S. D. M. 1997, ApJ, 490, 493 